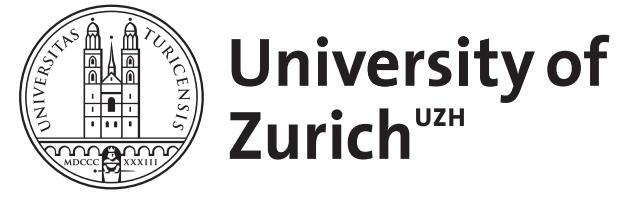

\title{
MultiNets: Web-based multilayer network visualization
}

Piškorec, Matija

DOI: https://doi.org/10.1007/978-3-319-23461-8_34

Posted at the Zurich Open Repository and Archive, University of Zurich

ZORA URL: https://doi.org/10.5167/uzh-206596

Conference or Workshop Item

Published Version

Originally published at:

Piškorec, Matija (2015). MultiNets: Web-based multilayer network visualization. In: European Conference on Machine Learning and Knowledge Discovery in Databases, n/a, 2015. s.n., 298-302.

DOI: https://doi.org/10.1007/978-3-319-23461-8_34 


\title{
MultiNets: Web-based multilayer network visualization
}

\author{
Matija Piškorec ${ }^{1}$, Borut Sluban ${ }^{2}$, and Tomislav Šmuc ${ }^{1}$ \\ 1 Rudjer Bošković Institute, Zagreb, Croatia, \\ matija.piskorec@irb.hr, \\ 2 Jožef Stefan Institute, Ljubljana, Slovenia \\ borut.sluban@ijs.si
}

\begin{abstract}
This paper presents MultiNets: a Javascript library for multilayer network visualization. MuLtiNets provides reusable HTML components with functions for loading, manipulation and visualization of multilayered networks. These components can be easily incorporated into any web page, and they allow users to perform exploratory analysis of multilayer networks and prepare publication quality network visualizations. MultiNets components are easily extendable to provide custom-based visualizations, such as embedding networks on geographical maps, and can be used for building complex web-based graphical user interfaces for data mining services that operate on multilayered networks and multirelational data in general.
\end{abstract}

Keywords: network visualization, multilayer networks, graph mining, network mining

\section{Introduction}

Network science is becoming an important multi-disciplinary research area including mathematics, physics, life sciences, social sciences, and computer science. Complex systems can often be represented as networks of interacting components, where each aspects of the system can be presented as an individual network, hence together forming a multi-aspect or multilayer network. The wide applicability of multilayer networks for solving and understanding different problem scenarios is gaining recognition in the scientific community $[2,6]$. In machine learning and data mining relevant research includes multilayer clustering [5], multi-network link prediction [11], mining heterogeneous networks [10] and meta-learning from network induced features [3].

Network visualizations offer an unique way to understand and analyze complex systems by enabling users to more easily inspect and comprehend relations between individual units and their properties [8]. The majority of programming languages offer libraries, modules and extensions for visualizing static networks. Many stand-alone programs for network visualization are available, for example Gephi $^{1}[1]$, Pajek $^{2}$, LaNet-vi $^{3}$, GraphViz $^{4}$, and ${ }^{*} \mathrm{ORA}^{5}$. Some extensions for data

\footnotetext{
1 http://gephi.github.io/

3 http://lanet-vi.soic.indiana.edu/

5 http://www.casos.cs.cmu.edu/projects/ora/
}

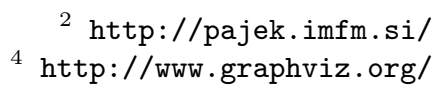


mining tools also exist: ORANGE offers the Network add-on ${ }^{6}$ for visualizing and analyzing networks, and KNIME has a network mining plug-in ${ }^{7}$. Recently, few stand-alone programs for multilayer network visualizations were developed, for example MuxViz [4] and Arena3D [9]. In comparison, there are far less tools for network visualization in web browsers, which is an oddity because webbased platforms are becoming increasingly common. Although some Javascript libraries offer basic support for network visualizations in web browsers, for example D3.js ${ }^{8}$, vis.js ${ }^{9}$, and sigma.js ${ }^{10}$, they are not tailored to support out-of-the box multilayered network visualizations. An exception is the HiveGraph application $^{11}$ which renders multilayer networks in a special hive plot layout [7].

This is why we developed MultiNeTs ${ }^{12}$ - a Javascript library that allows easy integration of interactive multilayer network visualization into any web page. MULTINETS consists of reusable HTML components that provide functions for visualization, loading, manipulation, filtering, and exporting of multilayer network data. It presents an unique and easy to use tool for the exploration of multilayer networks and multi-relational datasets in general. Because it is built using well known web technologies like HTML, CSS and Javascript, it is easily extendable, making it a perfect building block for complex web-based interfaces that use visualization as an aid in data analysis. Furthermore, the ubiquity of web browsers ensures that visualizations built with MultiNeTs will be accessible to the widest possible audience.

The source code of MultiNets is publicly available on the Github repository https://github.com/matijapiskorec/multinets.

\section{Implementation}

The MultiNets library is implemented as a set of Angular.js directives. Angular.js ${ }^{13}$ is a Javascript framework that supports the Model-View-Controller pattern (MVC) for building highly complex web-based user interfaces. Directives act as reusable components which users can easily include into their own web pages by using appropriate HTML elements. The main layout for presenting the multilayer network is based on the force layout from the D3.js Javascript library. In addition to visualization elements there are also elements responsible for selecting the layers of the network, loading network data from the server either statically or dynamically through REST API, loading network data from the user, and printing retrieved data. Users can easily extend the MultiNets library by defining custom directives that can generate visualizations tailored to specific domains or manipulate them in any desired way, as well as load and export networks in different data formats. To demonstrate this we implemented a special geographic layout that embeds the network of trade relations between countries on the World map. This layout also uses the D3.js library as a backbone

\footnotetext{
${ }^{6}$ http://orange.biolab.si/download ${ }^{7}$ https://tech.knime.org/network-mining 

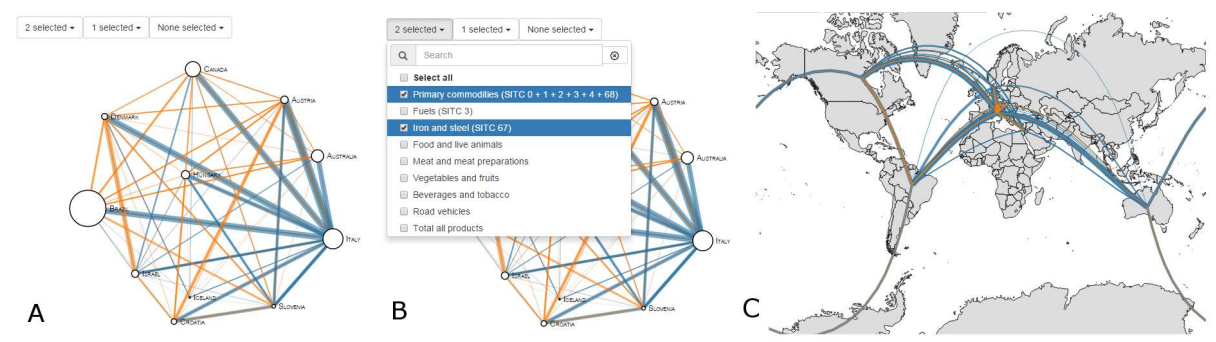

Fig. 1. Screenshots of MultiNets visualization of trade relations between countries, which is one of the use cases for multilayer network visualization. Panel $\mathbf{A}$ shows the visualization of the multilayer network of trade relations between countries. Sizes of the nodes are proportional to the population of each countries, colors of the connections correspond to various products and their thickness to the total trading volume. Other mappings from data to visual elements can be easily defined by modifying the existing components. Panel B shows the menu for selecting various aspects of the multilayer network, in this case the products traded between the countries. Multiple layers can be selected at once. Panel $\mathbf{C}$ shows geographical embedding of the given multilayer network. Users can extend MultiNeTs to provide similar embeddings for their specific domains.

for visualization, along with open source geographical data. The MultiNets library provides the following HTML elements:

- <force-layout $>$, <geo-layout $>$ Generate an interactive visualization of the multilayer network either as a force layout or embedded on the World map.

- <aspect-selector> Gives multi-choice menus for selection of various layers of networks.

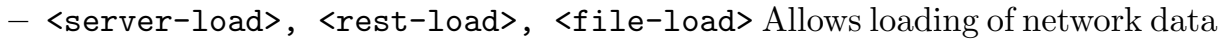
from server either statically or dynamically through REST API, and loading data from user.

- <network-dump> Outputs the retrieved network in text format.

\section{Summary}

Our motivating use case was to provide an easy to use web-based tool for exploratory data analysis of multilayered networks. By simple composition of HTML components users can build interfaces for comparison of different multilayered network datasets, exploratory data analysis, as well as prepare publication quality visualizations. Although MultiNets can visualize networks from any domain, the components of the library are easily extendable to support development of highly custom network visualizations tailored to specific domains. For example, the data mining community can use it to visualize large collection of multi-relational rules as an aid in various data analysis tasks. Also, 
in the biological domain users can build custom visualizations of gene regulatory networks or networks of metabolic pathways, and expose the visualization along with the data. In addition to providing easy to use exploratory analysis of different datasets, the high quality visualizations can be directly hosted on websites describing the research, or published as figures in articles and reports. The MultiNets library can also be extended with elements that expose meta information of the network dataset, including network based statistics like degree distribution of nodes, PageRank, betweenes, as well as many other statistics defined on the attributes of nodes or connections. Moreover, MultiNeTs components can be used for building web-based graphical user interfaces for advanced data mining services that operate on multilayered networks and multi-relational data in general.

\section{Acknowledgments}

This work was supported in part by the European Commission under the FP7 projects MULTIPLEX (grant no. 317532) and MAESTRA (grant no. 612944), Croatian Science Foundation (grant no. I-1701-2014) and by the Slovenian Research Agency programme Knowledge Technologies (grant no. P2-103).

\section{References}

1. Bastian, M., Heymann, S., Jacomy, M.: Gephi: An open source software for exploring and manipulating networks (2009)

2. Boccaletti, S., Bianconi, G., Criado, R., del Genio, C., Gmez-Gardees, J., Romance, M., Sendia-Nadal, I., Wang, Z., Zanin, M.: The structure and dynamics of multilayer networks. Physics Reports 544(1), 1-122 (2014)

3. Cheng, J., Adamic, L., Dow, P., Kleinberg, J., Leskovec, J.: Can cascades be predicted? WWW 2014 - Proceedings of the 23rd International Conference on World Wide Web pp. 925-935 (2014)

4. De Domenico, M., Porter, M.A., Arenas, A.: MuxViz: a tool for multilayer analysis and visualization of networks. Journal of Complex Networks (2014)

5. Gamberger, D., Mihelčić, M., Lavrač, N.: Multilayer clustering: A discovery experiment on country level trading data. In: Discovery Science. LNCS, vol. 8777, pp. 87-98. Springer (2014)

6. Kivelä, M., Arenas, A. Barthelemy, M., Gleeson, J.P., Moreno, Y., , Porter, M.A.: Multilayer networks. Journal of Complex Networks 2(3), 203-271 (2014)

7. Krzywinski, M., Birol, I., Jones, S.J.M., Marra, M.A.: Hive plots-rational approach to visualizing networks. Briefings in Bioinformatics 13(5), 627-644 (2012)

8. Rossi, L., Magnani, M.: Towards effective visual analytics on multiplex and multilayer networks. Chaos, Solitons \& Fractals 72(0), 68-76 (2015)

9. Secrier, M., Pavlopoulos, G.A., Aerts, J., Schneider, R.: Arena3D: visualizing timedriven phenotypic differences in biological systems. BMC Bioinform. 13, 45 (2012)

10. Sun, Y., Han, J.: Mining Heterogeneous Information Networks: Principles and Methodologies. Morgan \& Claypool Publishers (2012)

11. Zhang, J., Yu, P.S., Zhou, Z.H.: Meta-path based multi-network collective link prediction. In: ACM SIGKDD International Conference on Knowledge Discovery and Data Mining. pp. 1286-1295. KDD '14, ACM (2014) 